\title{
Energy Efficient Power Allocation for Distributed MIMO System
}

\author{
Qian Wang1,2, Yilong Yin ${ }^{1,2}$, Xiushan Nie ${ }^{1,2}$ \\ ${ }^{1}$ School of Computer Science and Technology, Shandong University of Finance and Economics, Jinan, China \\ ${ }^{2}$ School of Computer Science and Technology, Shandong University, Jinan, China \\ Email: qianwang@sdu.edu.cn, ylyin@sdu.edu.cn,niexiushan@163.com
}

How to cite this paper: Wang, Q., Yin, Y.L. and Nie, X.S. (2017) Energy Efficient Power Allocation for Distributed MIMO System. Int. J. Communications, Network and System Sciences, 10, 283-291. https://doi.org/10.4236/ijcns.2017.105B028

Received: May 22, 2017

Accepted: May 23, 2017

Published: May 26, 2017

\begin{abstract}
The rapid increasing demands of multi-media applications have busted the improvement of video transmission. By the increase of multimedia transmission, quality of experience $(\mathrm{QOE})$ has become the hot topic of the wireless networks' research. This paper proposes a QoE-aware resource allocation algorithm in MIMO-OFDM Networks. The power allocation estimation is formulated to maximize according to QoE by considering the video transmission rate and the subcarrier schemes under the constraint of minimum power. In this paper, we will analyses the the performance of video transmission from transmission rate at the application layer to the power allocation at the physical layer. From simulation results, we can see that the proposed subcarrier allocation algorithm maximizes the video QoE by minimizing the sum distortion of multi-users in MIMO networks with power constraints.
\end{abstract}

\section{Keywords}

QoE, Cross Layer Design, Resource Allocation, MIMO

\section{Introduction}

The rapid increasing demands of multi-media applications have busted the improvement of video transmission [1]. The development of physical (PHY) layer transmission schemes, such as Multiple Input and Multiple Output (MIMO) antennas, as well as multiple access schemes, e.g., Orthogonal Frequency Division Multiple Access (OFDMA), has obviously changed everyday life. The concept of quality of experience (QoE) means that we can evaluate the performance of communication networks and services under the subjective opinions from application users [2]. Many researchers have proposed the equations of QoS-QoE, which could reflect the relation between QoS and QoE parameter [3] [4] [5]. In 
order to optimize the resource allocation during media video transmissions in next LET, it is necessary to propose an improved resource allocation driven by QoE parameter [5].

Meanwhile, new video compression techniques have dramatically improved the compression efficiency of video codecs. The H.264/ AVC (H.264 Advanced Video Coding) [6] has been shown to save up to $55 \%$ of the bits compared to prior MPEG standards. Most recently, the HEVC (High Efficiency Video Coding) [7] is proposed to the improvement over the coding efficiency for the H.264. Along with the increasing demands on multimedia application, multimedia transmission is becoming one of the most popular services in future wireless networks [8] [9].

Recently, many researchers have been proposed some scheme for videos transitions over LET systems, which both PHY layer and APP layer parameter are considered to improve QoE of users [10] [11]. The performance of QoS-aware system can be improved considering the channel condition and RD at APP layer. In [11], an optimal subcarrier allocation for minimizing $\mathrm{RD}$ over MassiveMIMO systems is proposed, where video quality and channel capacity are considered. In [12], authors proposed perspectives and research challenges for QoE in video transmission over wireless networks.

In this paper, we focus on QoE-aware cross layer optimization in a multiple access environment. We investigate the resource allocation strategy by jointly considering the physical layer information and the application layer information. With the goal of QoE optimizing the overall system video performance, the PHY layer communication resources are allocated to the video users according to the demand of the multi-users.

The rest of the papers are written as following: Section II presents PHY layer and APP layer scheme, the same as the cross layer optimization. We will propose cross layer resource allocation in Section III, In Section IV Simulation results are showed, and in Section V, we will draw the conclusions.

\section{System Model}

We consider a MIMO OFDM network, where there are users transmitting their video streams to one access point (AP) through single hop route as shown in Figure 1.

\subsection{MIMO System Model}

In this Section, we consider a central-controlling cellular OFDMA multimedia communication networks. We set the users as $k=\{1,2,3, \cdots, K\}$ where to the base station went to communicate media. The $M$ is the number subcarrier. We can see $m=\{1,2, \cdots, M\}$. We can occupy a total frequency factors as $W(\mathrm{~Hz})$. All users adapt alphabet size of this modulation format, and adoptive QAM modulation is determined in the resource allocation scheme.

The user $k$ transmits the video packet with power $P_{k}$ under the maximum power constraint $P_{k}^{\max }$. The signal to interference plus noise ratio (SINR) for 


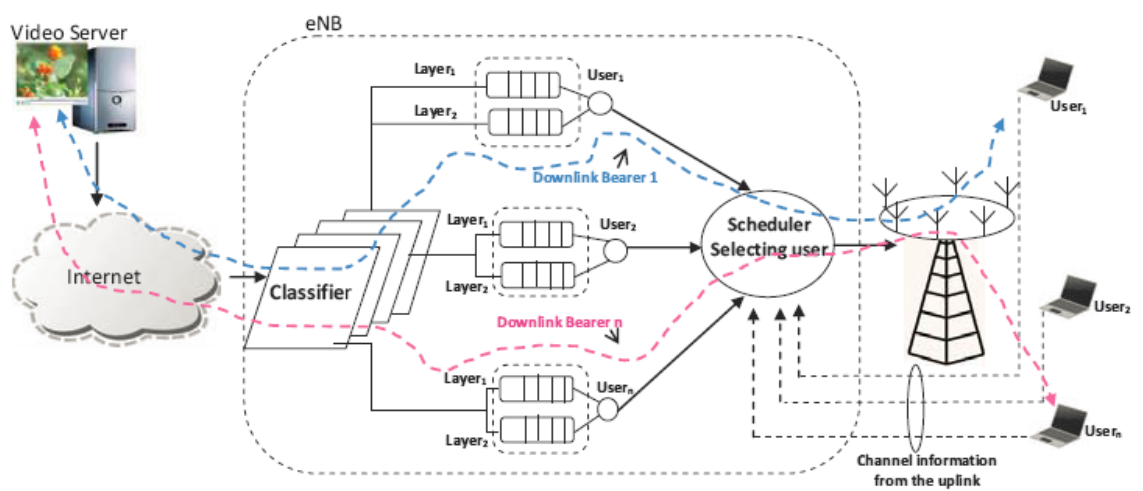

Figure 1. The structure of the proposed cross-layer transmission in MU-MIMO Communication systems.

user $\mathrm{k}$ is expressed as.

$$
\operatorname{SINR}_{k}=\frac{\gamma_{k} P_{k} G_{k}}{\Omega_{K}^{2}+\sum_{i \neq k} P_{j} G_{j}}
$$

where $\gamma_{k}$ represents the spreading gain and $G_{k}$ is the channel gain such as the large scale and small scale fading. $\Omega_{K}^{2}$ expresses the noise power of user $k$. The corresponding link capacity of user is given as

$$
C_{k}=B \log _{2}\left(1+S I N R_{k}\right)
$$

where $B$ is the channel bandwidth. To improve the quality of multimedia transmission, same assumption as made in [13] that the network works in the high SINR region. Therefore, the link capacity in (2) can be approximated as

$$
C_{k} \approx B \log _{2}\left(\frac{\gamma_{k} P_{k} G_{k}}{\Omega_{K}^{2}+\sum_{i \neq k} P_{j} G_{j}}\right)
$$

\subsection{QoE Evaluation of Multiuser}

QoE is an option-related metric which is important factor for future LTE systems. We use utility functions to describe QoE for multi-users in varied applications. The QoE of user $i$ can be expressed by $Q(\square)$ which is the quality function of systems. In this section, we use a QoE function, similar to [13]. It concludes a concave function of the rates. For a mutli-user $i$ under the same base station, corresponding to the rate $R_{i}$, the QoE of user $i$ can be described as:

$$
Q_{i}\left(R_{i}\right)=\frac{\ln \left(1+R_{i}\right)}{\ln \left(1+R_{i}^{r e q}\right)}
$$

where $R_{i}^{\text {req }}$ is t data rate of the $i$ user requesting.

\subsection{Video RD Characteristics}

Because the video is expressed as GOPs, this RD function is described under a GOP-by-GOP. Set $D_{i}^{k}(\sigma)$ is the rate distortion function of user $i$ in time slot $k$. For each GOP of user, the MSE distortion can be expressed as [14] [15] 


$$
D_{i}^{k}(\sigma)=\alpha_{i}+\frac{w_{i}}{B+\eta_{i}}
$$

where $\alpha_{i}, w_{i}$ and $\eta_{i}$ are constants, which will be jointly optimized by the PHY layer parameters by the cross layer design.

We put (4) into (5), then (5) can be written,

$$
D_{i}^{k}(\sigma)=\alpha_{i}+\frac{\frac{w_{i}}{w \bullet T_{s} / T_{0}}}{\sum_{m=1}^{M} \mu R_{i}^{m}\left(P_{i}^{m}, H_{i}^{m}\right)+\frac{\eta_{i}}{\eta \bullet T_{s} / T_{0}}}
$$

The QoE ignores the quality according to pricing $P_{i}\left(Q_{i}\right)$ from the users requirement in the MIMO-OFDMA network.

$$
\begin{aligned}
& \max . \quad \sum_{n=1}^{N} \sum_{i \in I} Q\left(R_{i}^{t_{n}}\right)-\gamma \Phi\left(R_{i}^{t_{n}}-R_{s}\right) \\
& \text { s.t. } \\
& \sum_{i=1}^{N} \frac{1}{m_{i} r_{i}} R^{i}\left(q_{i}, l_{f}\right) \leq R_{s}, \\
& \qquad \sum_{i=1}^{N} P_{i}\left(Q_{i}\right) \leq P_{b} \\
& q_{\min } \leq q_{i} \leq q_{\max }
\end{aligned}
$$

Note $\gamma$ is a quality parameter from the user. $\Phi(\square)$ is a quadratic equation for rate restrict. Thus, the $Q_{i}=Q^{i}\left(q_{i}, l_{f}\right)$ and $R^{i}\left(q_{i}, l_{f}\right)$ express the QoE quality parameter corresponding to the quantization value $q_{i}$. We assume the samelength frame rate $I_{f}$ and power constraint of $P_{b}$ for each user. We also assume the adaptive modulation parameter is $m_{i}$.

We can see (7) is convex and convert it into a standard form convex optimization problem [14] by modifying the optimization function,

$$
\min . \quad-\sum_{n=1}^{N} \sum_{i \in I} Q\left(R_{i}^{t_{n}}\right)-\gamma \Phi\left(R_{i}^{t_{n}}-R_{s}\right)
$$

We can apply employ the Karush-Kuhn-Tucker (KKT) framework to (8). The Lagrangian function $L(\bar{q}, \lambda, \bar{\mu}, \bar{\delta})$ is,

$$
\begin{aligned}
L(\bar{q}, \lambda, \bar{\mu}, \bar{\delta})= & -\sum_{i=1}^{N} n_{i}\left(\bar{\beta}_{i} q_{i}+\bar{\gamma}_{i}\right) \\
& +\lambda\left(\sum_{i=1}^{N} k_{i} e^{d_{i}\left(1-q_{i} / q_{\min }\right)}-R_{s}\right) \\
& +\sum_{i=1}^{N} \mu_{i}\left(q_{i}-q_{\max }\right)+\sum_{i=1}^{N} \delta_{i}\left(q_{\min }-q_{i}\right)
\end{aligned}
$$

where $\lambda, \mu_{i}, \delta_{i}, 1 \leq i \leq N$ are Lagrange multipliers, $\bar{\beta}_{i} \square e_{i} Q_{\max }^{i} Q_{t}\left(l_{f}\right) \beta_{i}$, $\gamma_{i} \square e_{i} Q_{\max }^{i} Q_{t}\left(l_{f}\right) \gamma_{i}$, We set $R_{\max }^{i}$ is the maximum rate of the $i^{\text {th }}$ video transmission. The quantity $k_{i}$ is as follow,

$$
k_{i} \square \frac{R_{\max }^{i}}{m_{i} r_{i}}\left(\frac{1-e^{-c_{t} l_{f} / t_{\max }}}{1-e^{-c_{t}}}\right)
$$


we obtain,

$$
-n_{i} \bar{\beta}_{i}-\lambda k_{i}\left(\frac{d_{i}}{q_{\min }}\right) e^{d_{i}\left(1-q_{i} / q_{\min }\right)}+\mu_{i}-\delta_{i}=0
$$

From (11), we can obtain the KKT complementary condition given as,

$$
\lambda \sum_{i=1}^{N} k_{i} e^{d_{i}\left(1-q_{i} / q_{\min }\right)}-R_{s}=0
$$

From (12), we set $\mu_{i}=0$ and $\delta_{i}=0$, the Lagrangian multiplier $\lambda^{*}$ can be expressed as,

$$
\lambda^{*}=-\frac{q_{\min }}{R_{s}}\left(\sum_{j=1}^{N} \frac{\bar{\beta}_{j} n_{j}}{d_{j}}\right)
$$

We substitute the above expression for the optimal quantization parameter $q_{i}^{*}$ and then,

$$
\begin{aligned}
q_{i}^{*} & =\frac{q_{\min }-q_{\min } \ln \left(\frac{q_{\min } \bar{\beta}_{i i} m_{i} r_{i}}{\lambda^{*} k_{j} d_{j}}\right)}{d_{j}} \\
& =\frac{q_{\min }-q_{\min } \ln \left(\frac{R_{s}}{k_{i}} \frac{n_{i} \beta_{i}\left(d_{i}\right)^{-1}}{\sum_{j=1}^{N} n_{j}\left(d_{i}\right)^{-1}}\right)}{d_{j}}
\end{aligned}
$$

The above expression can obtain the optimal quantization parameter $q_{i}^{*}$ for resource allocation. The (14) has low computational complexity scheme for optimal video transmission for both unicast and multicast surrounding. This proposed joint power and subcarrier allocation algorithm in MIMO OFDM systems enables us both to overcome the challenge of full CSI. We can get optimal subcarrier and power allocation by iterative as Table 1 .

Table 1. Iterative Subcarrier and Power Allocation.

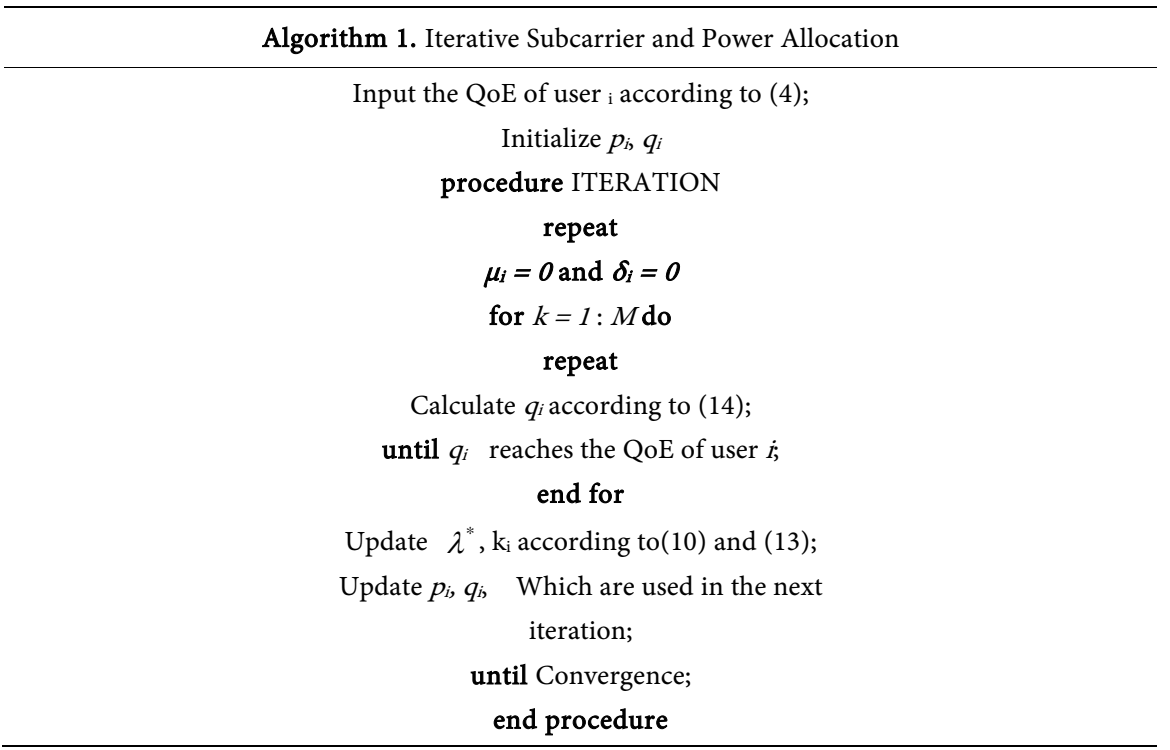




\section{Simulation Results}

In this section, we simulate the performance results for the MU-MIMO OFDM systems with an amount of 32 subcarriers. And the system power constrain is $-120 \mathrm{~dB} / \mathrm{Hz}$.. Under the given power constraint in each bandwidth, power can be expressed as $\mathrm{Pt}=200 \mathrm{~mW}$, of which has each bandwidth of $100 \mathrm{kHz}$. We can denote the path-loss con-strain is $\gamma=2.45$. Moreover, $\alpha$ is a Rayleigh fading variable. For each time slot $i$ the optimization system can be automatically transferred.

In simulation, the aim of the allocations is to be outputted to the rate and subcarrier allocation corresponding to the number of users. Figure 2 describe a set of simulate results. From Figure 2, we can see, with cross layer design, requirement of the packet loss in APP layer varying with the channel fading. Hence the power control coefficients can be made independent of frequency and their effect on the data rate obtained by QAM. The valid PSNR is about $40 \%$ toward to SER. In one word, to obtain the target PSNR under 16QAM, the rate allocation is bigger than the result of the other OFDMA system.

When the transmission power is decrease, the result can be shown will be made. This simulation can be shown in Figure 3 Figure 4, where the PSNR is applied under this value. When the value of power is decrease, the result can be shown will be made. This simulation to can be shown in Figure 3, where the PSNR is applied $\mathrm{f}$ under this value. That is to say 8 users computing for resources which here are 64 sub-carriers are obtained.

Our simulation results show that the optimal cross-layer design is achieved highly performance according to the curve of QoE. Compared to a resource algorithm using either only PHY layer or only APP layer, the cross layer optimization significantly improved the performance of the sys-tem. It is also achieved highly throughout and low delay in this numerical results.



Figure 2. Average media video quality vs. number of users. 


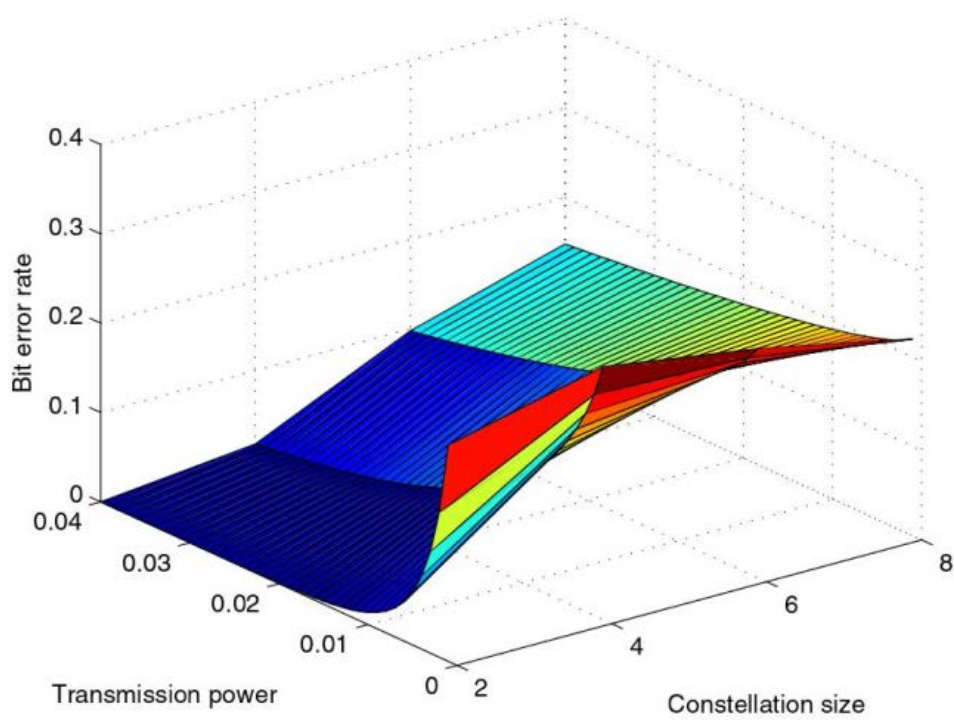

Figure 3. Bit error rate under different power and constellation size.

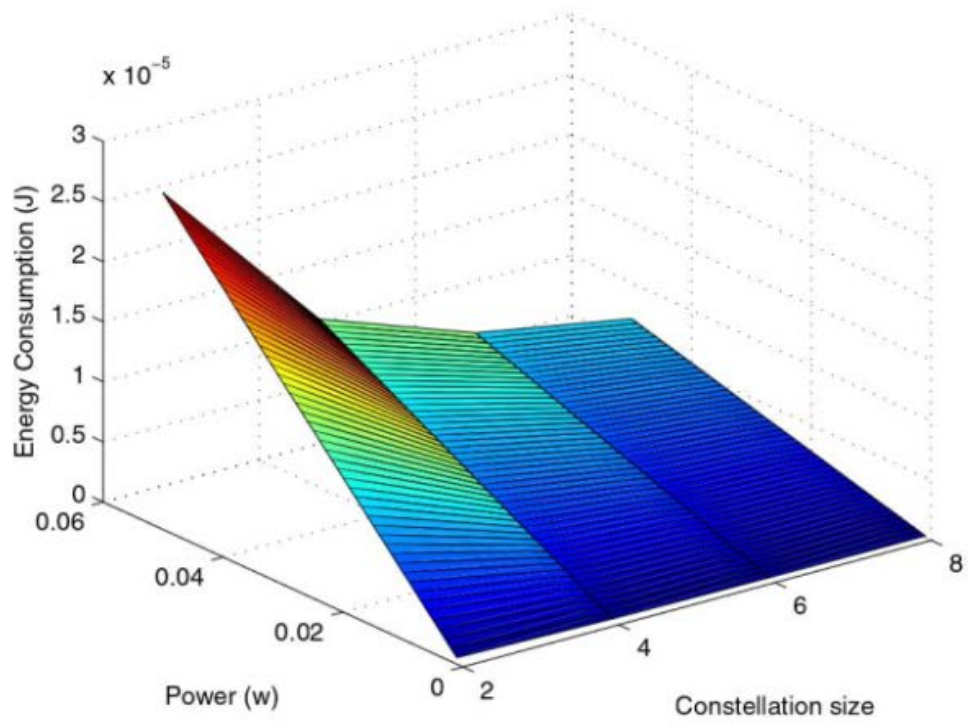

Figure 4. Energy consumption with different power and constellation size.

\section{Conclusion}

In this paper, aiming to improve the quality of multimedia transmission while satisfying the QoE requirements, we propose an optimal subcarrier allocation by jointly considering the video coding rate and the available power resource. We jointly analyses the effects on the performance of multimedia transmission from video coding rate at the application layer as well as the power control at the physical layer. This proposed joint power and subcarrier allocation algorithm in MIMO OFDM systems enables us both to overcome the challenge of full CSI to make minimum of the distortion of each users under delay and power constraints. Simulation results show that the proposed optimal power allocation algorithm improves the multimedia transmission quality considerably through the comparison with the resource allocation algorithms only use a single layer of in- 
formation.

\section{Acknowledgements}

This work was supported in part by the National Natural Science Foundation of China (No. 61371109, 61671274) and NSFC Joint Fund with Guangdong under Key Project (No. U1201258) and Shandong Natural Science Funds for Distinguished Young Scholar (No. JQ201316).

\section{References}

[1] Cisco (2015) Cisco Visual Networking Index: Glo- bal Mobile Data Traffic Forecast Update 2014C2019 White Paper. Tech. Rep.

[2] Zhou, L., Yang, Z., Wen, Y. and Rodrigues, J. (2013) Distributed Wireless Video Scheduling with Delayed Control Information. IEEE Transactions on Circuits Syst. Video Technol., 24, 889-901. https://doi.org/10.1109/TCSVT.2013.2291311

[3] Wu, J., Cheng, B., Yuen, C., Shang, Y. and Chen, J.-L. (2015) Distortion Aware Concurrent Multipath Transfer for Mobile Video Streaming in Heterogeneous Wireless Networks. IEEE Trans. Mobile Comput., 14, 345-360. https://doi.org/10.1109/TCSVT.2013.2291311

[4] Chan, Y., Cosman, P. and Milstein, L. (2008) A Multiple Description Coding and Delivery Scheme for Motion-Compensated Fine Granularity Scalable Video. IEEE Transactions on Image Processing, 17, 1353-1367. https://doi.org/10.1109/TIP.2008.925300

[5] Bansal, G., Hossain, M., Bhargava, V. and Le-Ngoc, T. (2013) Subcarrier and Power Allocation for Ofdma-Based Cognitive Radio Systems with Joint Overlay and Underlay Spectrum Access Mechanism. IEEE Trans. Veh. Technol., 62, 1111-1122. https://doi.org/10.1109/TVT.2012.2227856

[6] “International Telecommunication Union AAP An-nouncement No. 09". http://www.itu.int/dms pubaap/01/T0101000F09.htm.

[7] Zhou, L., Yang, Z., Wen, Y. and Rodrigues, J. (2013) Distributed Wireless Video Scheduling with Delayed Control Information. IEEE Trans. Circuits Syst. Video Technol., 24, 889-901. https://doi.org/10.1109/TCSVT.2013.2291311

[8] Mukhtar H., Al-Dweik A., Al-Mualla M., et al. (2015) Low Complexity Power Optimization Algorithm for Multimedia Transmission over Wireless Networks. IEEE Journal of Selected Topics in Signal Processing, 9, 113-124. https://doi.org/10.1109/JSTSP.2014.2331915

[9] Kuo W.H., Kaliski R., and Wei, H.Y. (2015) A QoE-based Link Adaptation Scheme for H. 264/SVC Video Multicast over IEEE 802.11. IEEE Transactions on Circuits and Systems for Video Technology, 25, 812-826. https://doi.org/10.1109/TCSVT.2014.2363741

[10] Su, G.M., Su, X., Bai, Y., et al. (2016) QoE in Video Streaming over Wireless Networks: Perspectives and Research Challenges. Wireless Networks, 22, 1571-1593. https://doi.org/10.1007/s11276-015-1028-7

[11] Vosoughi, A., Cosman, P.C. and Milstein, L.B. (2015) Joint Source-Channel Coding and Unequal Error Protection for Video Plus Depth. IEEE Signal Processing Letters, 22, 31-34. https://doi.org/10.1109/LSP.2014.2346739

[12] Reichl, P., Egger, S., Schatz, R. and Alconzo, A.D. (2010) The Logarithmic Nature of QoE and the Role of the Weber-Fechner Law in QoE Assessment. Proc. IEEE Int. Conf. Commun. 1-5. 
[13] Stuhlmuller, K., Farber, N., Link, M. and Girod, B. (2000) Analysis of Video Transmission over Lossy Channels. IEEE Journal on Selected Areas in Communications, 18, 1012 -1032. https://doi.org/10.1109/49.848253

[14] Kim, H., Annavajjala, R., Cosman, P. and Milstein, L. (2010) Source-Channel Rate Optimization for Progressive Image Transmission over Block Fading Relay Channels. IEEE Transactions on Communications, 58, 1631-1642. https://doi.org/10.1109/TCOMM.2010.06.080203

[15] Saki, H. and Shikh-Bahaei, M. (2015) Cross-Layer Resource Allocation for Video Streaming over OFDMA Cognitive Radio Networks. IEEE Trans. on Multimedia, 17, 333-345. https://doi.org/10.1109/TMM.2015.2389032

Submit or recommend next manuscript to SCIRP and we will provide best service for you:

Accepting pre-submission inquiries through Email, Facebook, LinkedIn, Twitter, etc. A wide selection of journals (inclusive of 9 subjects, more than 200 journals)

Providing 24-hour high-quality service

User-friendly online submission system

Fair and swift peer-review system

Efficient typesetting and proofreading procedure

Display of the result of downloads and visits, as well as the number of cited articles Maximum dissemination of your research work

Submit your manuscript at: http://papersubmission.scirp.org/

Or contact ijcns@scirp.org 